\title{
Electron correlation effects in attosecond photoionization of $\mathrm{CO}_{2}$
}

\author{
Andrei Kamalov, ${ }^{1,2, *}$ Anna L. Wang $\odot,{ }^{1,3}$ Philip H. Bucksbaum $\odot,{ }^{1,2,3}$ Daniel J. Haxton, ${ }^{4}$ and James P. Cryan $\odot^{1,5, \uparrow}$ \\ ${ }^{1}$ Stanford PULSE Institute, SLAC National Accelerator Laboratory, Menlo Park, California 94025, USA \\ ${ }^{2}$ Department of Physics, Stanford University, Stanford, California 94305, USA \\ ${ }^{3}$ Department of Applied Physics, Stanford University, Stanford, California 94305, USA \\ ${ }^{4}$ KLA Corporation, Milpitas, California 95035, USA \\ ${ }^{5}$ Linac Coherent Light Source, SLAC National Accelerator Laboratory, Menlo Park, California 94025, USA
}

(Received 23 April 2020; accepted 17 July 2020; published 26 August 2020)

\begin{abstract}
A technique for measuring photoionization time delays with attosecond precision is combined with calculations of photoionization matrix elements to demonstrate how multielectron dynamics affect photoionization time delays in carbon dioxide. Electron correlation is observed to affect the time delays through two mechanisms: autoionization of molecular Rydberg states and accelerated escape from a continuum shape resonance.
\end{abstract}

DOI: 10.1103/PhysRevA.102.023118

\section{INTRODUCTION}

Photoionization is a basic quantum scattering process involving the rearrangement of degrees of freedom in the total system. In the time domain, this is described by an incoming photon wave packet that couples to outgoing electron wave packets (EWPs) in the final-state cation channels. The term "photoionization time delay" refers to the time required for a photoionized EWP to propagate out of the electric potential of the residual cation. It may be defined semiclassically as the difference in time required to propagate a photoelectron from its birth location to a detector position, compared to some reference [1-3]. The bound electron loses kinetic energy as it escapes the Coulomb potential; moreover, kinetic energy may be redistributed through Coulomb and exchange scattering with other electrons. This redistribution dynamically modifies the ionic potential, resulting in dynamics on the attosecond timescale. Although the asymptotic state of the total system (cation plus ionized electron) is easily understood in a single-electron picture, this picture may break down when the electron has not yet escaped into the asymptotic region for detection [4]. The photoionization time delays are influenced by modifications of the effective potential experienced by the ionized electron [5,6] and therefore may be used to extract information about the temporal evolution of the electronelectron interactions near the nuclei.

Recent advances in the production of attosecond laser pulses have enabled direct probing of the two-photon photoionization time delay, $\tau^{(2)}$, using two-color interferometric techniques [5]. Under ideal conditions, the delay described above for single-photon ionization can be extracted from the measured two-photon photoionization delay [1]. In general, this extraction is not possible because the interaction with the

\footnotetext{
*andrei.kamalov@gmail.com

†jcryan@slac.stanford.edu
}

IR dressing field couples the various partial wave contributions $[4,7]$. However, the two-photon photoionization delay is still a valid observable, and can be described as the first derivative of the phase of a transition matrix element. Moreover, this two-photon delay contains information about the photoionization dynamics of a system. We can infer the quantum dynamics of the photoionization process by combining this measured quantity with theoretical modeling [1-3,5,6,826]. Therefore, for the remainder of the paper we will use the term photoionization time delay to refer to the experimentally accessible, two-photon photoionization time delay.

Previous measurements of photoionization time delays have made use of the sensitivity of photoionization time delay measurements to time-dependent changes of the ionic potential to gain a deeper understanding of electron correlations in shake-up ionization [6] and atomic autoionization [21-25]. This work combines measurements of the photoionization time delays with numerical calculations of photoionization probability amplitudes to demonstrate how multielectron dynamics affect ionized EWPs in a molecular system. In our comparison we find time-delay signatures of multielectron effects in the vicinity of autoionizing and molecular shape resonances. The enhancement of electron correlation effects near molecular shape resonances was previously considered by Siggel et al., who showed that the photoelectron angular distribution can be sensitive to multielectron channel coupling phenomena, whereas the channel-resolved cross section is much less sensitive [27]. The effect that electron-electron interactions would have on the interpretation of photoionization time delay measurements near molecular shape resonances has yet to be considered in the literature.

Carbon dioxide $\left(\mathrm{CO}_{2}\right)$ provides a particularly striking example of multielectron dynamics in molecular photoionization [28-31]. Straightforward close-coupling expansions require 96 individual cation configurations to reproduce the experimental cross section [31], far more states than are energetically available as photoionization channels. The virtual excitations of the closed cationic channels correspond to 


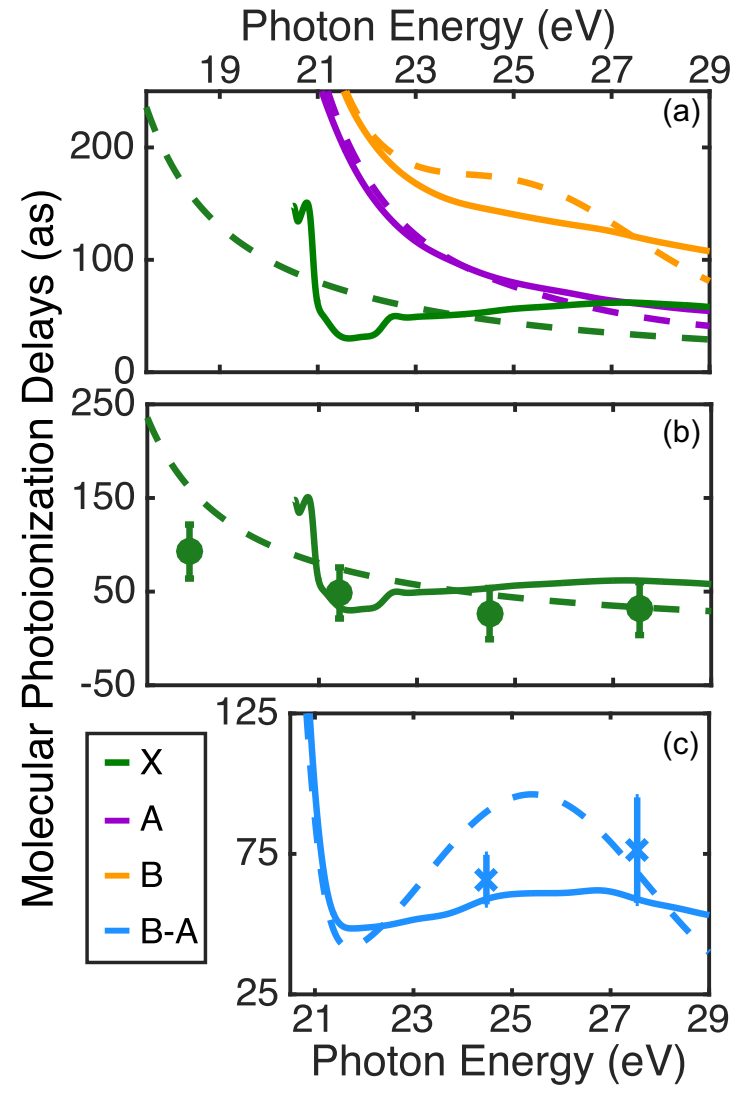

FIG. 1. Two-photon molecular photoionization time delays $\left[\tau_{P I}\right.$ in Eq. (4)] for unaligned $\mathrm{CO}_{2}$. Panel (a) shows our calculations done in two approximations (see text) - the independent channel approximation (dashed) and coupled-channel method (solid). The calculations are shown as a finite difference derivative of the photoionization scattering phase, as defined in Eq. (3), incoherently summed across all molecular orientations and electron emission angles to match experimental conditions. Panel (b) shows measured and calculated results for the $X^{2} \Pi_{g}$ channel. Panel (c) shows results for the $B^{2} \Sigma_{u}{ }^{+}$channel referenced to the $A^{2} \Pi_{u}$ channel. In all panels, the error bars represent the $\pm 2 \sigma$ confidence level.

multiple-electron excitations of the molecular system, which affect the magnitude and phase of the EWPs escaping into the energetically open channels. In this work we measure photoionization time delays for $\mathrm{CO}_{2}$ and demonstrate that agreement with calculated time delays is contingent upon including electron correlation effects in the calculation.

Figure 1 shows the measured two-photon molecular photoionization time delays for ionization processes leading to the $X^{2} \Pi_{g}, A^{2} \Pi_{u}$, and $B^{2} \Sigma_{u}{ }^{+}$cationic states of $\mathrm{CO}_{2}$. These time delays are obtained using a two-color, multipath interference technique, which overlaps a weak infrared (IR) laser pulse, with photon energy $\hbar \omega$, and an XUV frequency comb with spectral peaks separated by $2 \hbar \omega$. This is a variant of the RABBITT (reconstruction of attosecond bursts by inference in twophoton transitions) technique $[32,33]$. The XUV frequency comb appears in the time domain as an attosecond pulse train (APT), whose pulses provide the temporal resolution needed to measure EWP delays. Ionization of the $\mathrm{CO}_{2}$ target by only the APT produces a series of peaks spaced by $2 \hbar \omega$, as shown

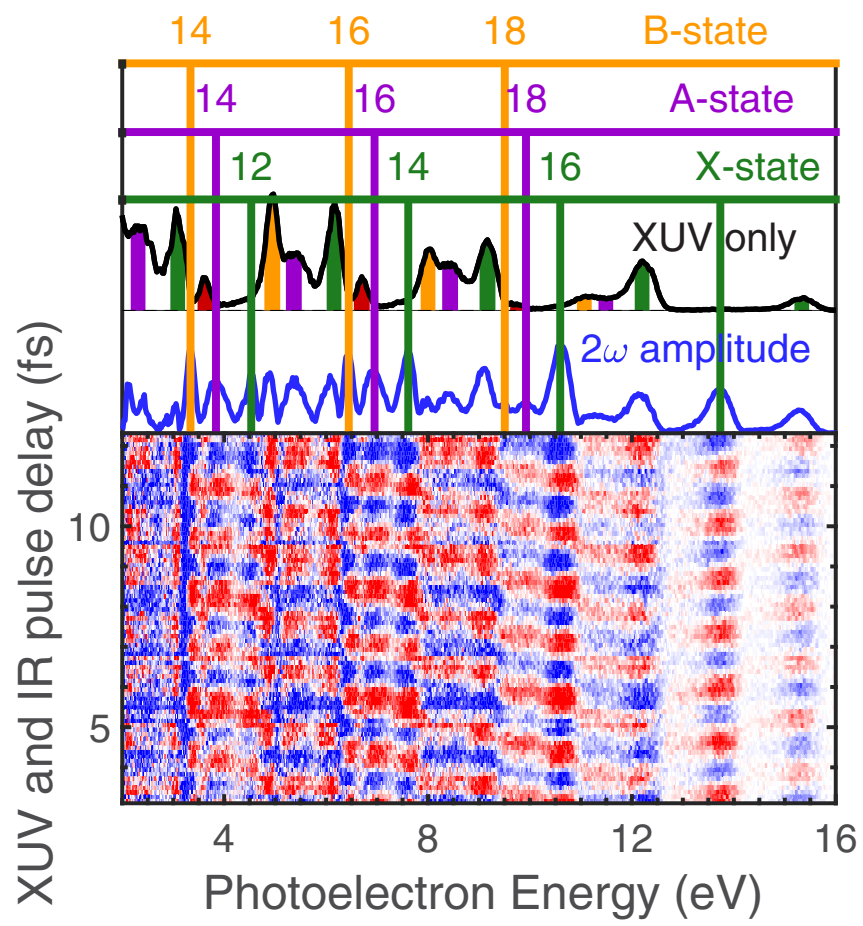

FIG. 2. Two-color photoelectron spectrogram of $\mathrm{CO}_{2}$. The upper panel shows the photoelectron spectrum recorded with only the XUV APT present. The colored areas denote the photoionization channel responsible for each spectral feature, and are color coded to match the state labels. The red areas in the XUV-only panel show features associated with the $C^{2} \Sigma_{g}$ state. The middle panel shows the amplitude of the $2 \omega$ oscillation retrieved from the Fourier transform of the lower panel. The lower panel shows a subset of the measured photoelectron spectrogram and demonstrates clear $2 \omega$ oscillation. To make the oscillations more clear, we have subtracted the delay-averaged photoelectron spectrum. All features in this panel have been assigned to either a harmonic or sideband feature of a $\mathrm{CO}_{2}{ }^{+}$final state.

in the top panel of Fig. 2. The weak IR field induces twophoton transitions, producing "sideband" features between adjacent spectral features in the XUV frequency comb. Scanning the phase between the IR field and the APT results in a modulation of the sideband features as seen in the bottom panel of Fig. 2. This modulation occurs at twice the IR laser frequency and can be described by

$$
\mathrm{SB}_{2 n}(\tau) \propto \cos \left[2 \omega \tau+\phi_{2 \omega}(2 n)\right],
$$

where $\mathrm{SB}_{2 n}$ is the yield of a sideband peak as a function of $\tau$, the relative delay between the IR and XUV fields, and $\phi_{2 \omega}(2 n)$ is a phase offset in the sideband modulation that varies with sideband order $(2 n)$. The sideband modulation is caused by interference between two different two-photon ionization pathways, involving the absorption of an XUV photon and subsequent absorption or emission of an IR photon [1,32-34].

The sideband phase offset can be parsed into two contributions:

$$
\phi_{2 \omega}(2 n)=\Delta \phi_{\mathrm{XUV}}+\Delta \phi,
$$


where $\Delta \phi_{\mathrm{XUV}}$ describes the spectral phase difference between consecutive features in the XUV frequency comb that contribute to the sideband peak. $\Delta \phi$ describes the phase difference between two-photon ionization matrix elements leading to the sideband feature $[1,33]$. The RABBITT technique was originally developed to characterize APTs by extracting the first term in Eq. (2) [32,33]. Subsequent work has focused on the latter quantity in Eq. (2) to extract a delay for the two-photon ionization process $\left(\tau^{(2)}\right)$ :

$$
-\frac{\tau^{(2)}}{\hbar}=\frac{\Delta \phi}{2 \hbar \omega} \approx \frac{\partial \phi}{\partial E}
$$

where $\phi$ is again the phase of the two-photon matrix element. The approximation in the latter half of Eq. (3) holds for the case when $\phi(E)$ changes slowly over the energy interval between the harmonic features. For a structured continuum this approximation breaks down, in which case the two-photon delay is given by the average value of $\partial \phi / \partial E$ over this same energy interval.

In many cases, the two-photon delay in Eq. (3) can be separated into a measurement induced (or continuum-continuum) contribution $\left(\tau_{\mathrm{cc}}\right)$ that simply depends on the energy of the outgoing electron $\left(\epsilon=E_{2 n}-I_{p}\right.$, where $\left.E_{2 n}=2 n \hbar \omega\right)$, and a potential-dependent term $\left(\tau_{P I}\right)[1,2,4,7,35]$ :

$$
\tau^{(2)}\left(E_{2 n}\right) \approx \tau_{P I}\left(E_{2 n}\right)+\tau_{\mathrm{cc}}(\epsilon),
$$

as shown in the Appendix. When the system is spherically symmetric and the ionization process is dominated by a single angular momentum partial wave, the potential dependent term can be shown to approximate the single-photon photoionization time delay $[1,35]$. In this limit, the two-photon interferometric technique has been used to investigate photoionization time delays for different continuum channels in atomic targets $[2,15,36]$, relative photoionization time delays between atomic targets $[12,13]$, and resonant processes in atomic photoionization [21-25].

The interpretation of the interferometric measurements is more challenging for molecular systems. Molecular targets often have several accessible cationic states that lead to substantial overlap of features in the photoelectron spectra (spectral congestion) [37]. Isolating the contributions from different final states in the spectrum requires high resolution measurements. Moreover, the partial-wave expansion of outgoing photoelectron wave packets can contain a large number of coherent contributions. The coherent summation over partialwave contributions means that molecular photoionization interference measurements are not easily parsed to recover the single photon photoionization delay. Nevertheless, Huppert et al. recently observed the effect of a molecular shape resonance on the measured photoionization time delays in $\mathrm{N}_{2} \mathrm{O}$ [18]. Prior to this work, Haessler et al. observed a "complex resonance" in the photoionization of low-lying vibration states of the $X$ channel of $\mathrm{N}_{2}$ [17]. Vos et al. were able to study the stereo Wigner time delay in carbon monoxide averaged over a number of dissociative states of the $\mathrm{CO}^{+}$cation [19]. Due to the excellent kinetic-energy resolution afforded by the magnetic bottle spectrometer, we are able to resolve the sideband oscillations for three final-state channels in $\mathrm{CO}_{2}$ (middle panel of Fig. 2) to produce the measurements of
$\tau_{P I}\left(E_{2 n}\right)$ shown in Fig. 1. Further details of the measurement procedure are given below in the Methods section.

\section{OBSERVATION OF CONTINUUM CHANNEL COUPLING IN PHOTOIONIZATION TIME DELAYS}

In order to understand the dynamics captured in the time delay measurements shown in Fig. 1, we compare these data with predicted delays calculated using an implementation [38-46] of the complex Kohn variational method [47-53] for photoionization [54-63] and electron-molecule scattering [64-73]. The two-photon molecular photoionization time delays $\left(\tau_{P I}\right)$ are calculated in two different levels of approximation and then averaged over molecular orientation and outgoing electron direction, consistent with the measurement scheme used in the experiment (see the Appendix). The independent channel approximation considers the scattering in each continuum channel separately. The coupled-channel method uses fully coupled continuum states, thereby allowing electrons originally produced in one ionization channel to interact with the residual ionic core to produce different final-state configurations. More details of the complex-Kohn calculation are given below in the Methods section and in the Supplemental Material (SM) [74].

The independent channel calculations [dashed lines in Fig. 1(a)] for the $X^{2} \Pi_{g}$ and $A^{2} \Pi_{u}$ channels display traditional Coulombic behavior: monotonically increasing photoionization delay with decreasing photon energy [2]. The $B^{2} \Sigma_{u}{ }^{+}$channel exhibits an increased photoionization delay time around a photon energy of $25 \mathrm{eV}$, which is a signature of a weak shape resonance $[7,18,75]$ that has been observed in the $\mathrm{CO}_{2}$ absorption spectrum [27]. The interchannel coupling alters the predicted photoionization time delays [solid lines in Fig. 1(a)]. At photon energies above $20 \mathrm{eV}$, coupling among the continuum channels results in a decrease in the photoionization time delay for the $B^{2} \Sigma_{u}{ }^{+}$state in the vicinity of the shape resonance feature. Electron interactions induce coupling between the $B$-state continuum and other continuum channels while the wave packet is trapped in the vicinity of the ionic core. The coupling is strongest between the $B$ - and $C$-state channels. This coupling provides additional pathways for electrons to escape the shape resonance and thus lowers the photoionization delay times for the $B^{2} \Sigma_{u}{ }^{+}$ state. Consequently, there are increases in the photoionization time delays for the other channels that the $B$-state continuum couples to, namely the $X$-, $A$-, and $C$-state channels.

Figure 1(c) compares our measured photoionization delays in the vicinity of the $B$-state shape resonance to the theoretical predictions of both models. It shows that these measured photoionization delays are consistent with the coupled-channel theory, whereas there is a disagreement with the singlechannel predictions. These time-domain measurements show how electron correlation dynamics can accelerate the escape of the photoelectron from the molecular potential, and thus lower the photoionization time delay.

\section{MOLECULAR AUTOIONIZING STATES}

Figure 1(b) shows the measured photoionization time delays for the $X^{2} \Pi_{g}$ channel along with the single-channel 
and coupled-channel calculations. For photon energies above $20 \mathrm{eV}$, the single-channel and coupled-channel calculations predict similar photoemission delay values, which are consistent with our measurements. Below $20 \mathrm{eV}$, the measured photoionization delay is shorter than the single-channel model predicts.

The sideband feature at $18.4 \mathrm{eV}$ originates from the interference between absorption pathways involving features of the XUV frequency comb at $16.8 \mathrm{eV}$ and $19.9 \mathrm{eV}$. The lower energy comb feature completely overlaps two autoionizing Rydberg series converging to the $A^{2} \Pi_{u}$ and $B^{2} \Sigma_{u}{ }^{+}$ionization thresholds (17.59 and $18.07 \mathrm{eV}$, respectively), while the higher-energy comb feature overlaps with a smooth continuum. Previous works have shown that the presence of these resonant absorption features can have a significant effect on the measured two-photon delay [4,21-26]. A majority of the previous experimental work (Refs. [21-24]) focused on isolated resonances in atomic systems. Beaulieu et al. studied an isolated autoionizing resonance in multiphoton ionization of a chiral molecular system [26]. In the present work, the XUV frequency comb overlaps many resonant features, similar to the recent work of Barreau et al., who demonstrated a disentangling of the complex behavior of two-photon photoionization phases in overlapping autoionizing states in atomic neon [25]. The results of Barreau et al. show various resonant contributions are important for understanding the measurement.

Observing phenomena such as autoionization, which typically have lifetimes longer than the interpulse spacing of our XUV pulse train $(1.35 \mathrm{fs})$, requires additional considerations in the analysis. Since each attosecond burst in the APT has a coherent relationship to the neighboring pulses, when timedependent phenomena extend beyond the interpulse spacing, the processes induced by a single burst in the train interferes with the signal generated by the adjacent pulses. This interference leads to a burst-dependent photoemission time delay, i.e., the peak emission time is the point where all previous photoemission processes interfere constructively. Such a modulation (burst-to-burst) of the photoemission time delay leads to a variation of the phase across a sideband. This revelation is the motivation behind the development of the Rainbow RABBITT technique [21,24]. Applying this type of analysis, the derivative of $\phi_{2 \omega}$ with respect to the photoelectron kinetic energy for each sideband feature is shown in Fig. 3.

Most of the sideband features display a very minimal slope, which becomes slightly negative at lower photoelectron energies. This behavior is due to the harmonic chirp (or femtochirp) present in the APT [76]. However, the $X$-channel feature at $18.4 \mathrm{eV}(4.2 \mathrm{eV}$ photoelectron energy, marked as SB 12 in Fig. 3) displays an anomalous slope compared with the other channels, and targets.

To better understand the effect of the resonant states we show the measured amplitude and phase of the $2 \omega$ oscillation across this sideband feature in Fig. 4. We have corrected the measured phase shown in Fig. 4 to account for any harmonic chirp from the XUV pulse train. This was done by subtracting a linear phase offset measured in the krypton data set shown in Fig. 3. The small feature on the low-energy side of the measured amplitude, near $18.3 \mathrm{eV}$, is due to vibrational excitation of the $X$-state cation.

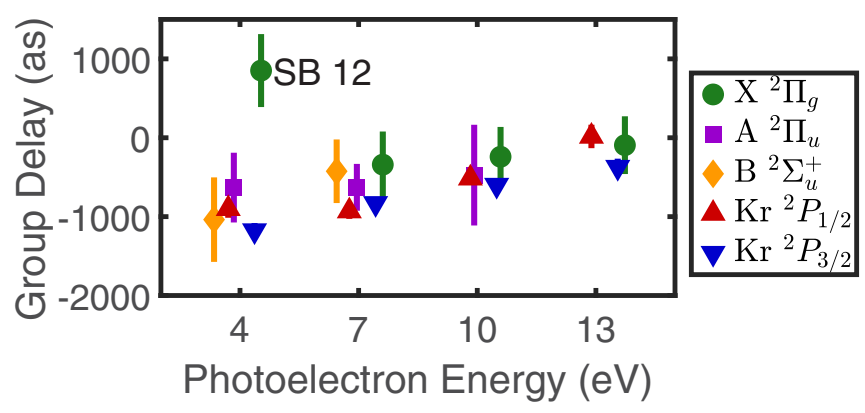

FIG. 3. Group delay $\left(-\hbar \frac{\partial \phi_{2 \omega}}{\partial E}\right)$ taken across a sideband feature as a function of photoelectron kinetic energy for $\mathrm{CO}_{2} X^{2} \Pi_{g}$ (circle), $A^{2} \Pi_{u}$ (square), $B^{2} \Sigma_{u}{ }^{+}$(diamond) channels, $\mathrm{Kr}^{2} P_{1 / 2}$ channel (upwards pointing triangle), and $\mathrm{Kr}^{2} P_{3 / 2}$ channel (downwards triangle).

Ideally we would compare this measurement using the method developed in the Appendix. The coupled-channel complex-Kohn calculations should be able to accurately describe autoionizing resonances. However, for high-lying autoionizing resonances, the convergence of the complex Kohn method is difficult due to the need for a large number of basis functions and the separable exchange approximation that is made. We have found that our implementation does not reliably estimate the structures in the $\mathrm{CO}_{2}$ absorption spectrum between 15 and $18 \mathrm{eV}$. Therefore, we cannot compare the multichannel theory with the data in Fig. 4, and we have excluded the multichannel calculations in Fig. 1(b) where these autoionizing states influence the measurement.

Instead we use the analytical model developed by JiménezGalán et al. [77], and described in the Appendix. Similar to Ref. [25], we assume that radiative transitions from discrete components of the autoionizing resonance to the continuum are negligible. With this simplification the variation in the

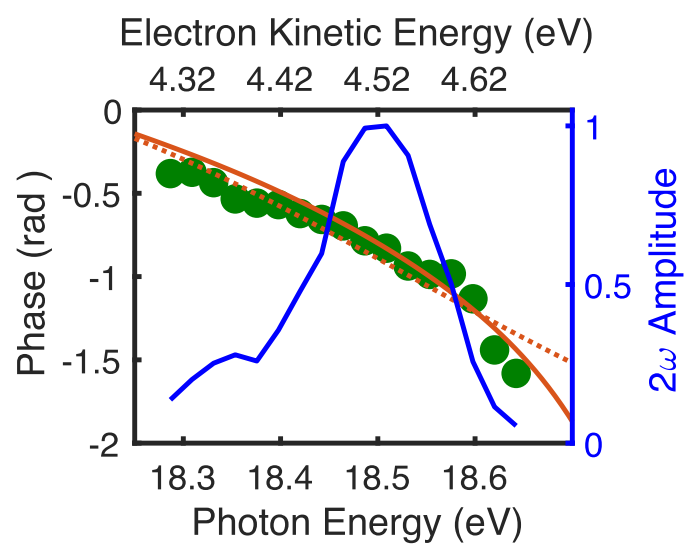

FIG. 4. Measured amplitude ( $A_{2 \omega}$ blue line, right axis) and phase ( $\phi_{2 \omega}$ full circle, left axis) of the $2 \omega$ oscillation as a function of photoelectron (photon) energy for the $X$-state sideband feature at $18.4 \mathrm{eV}$. We compare these measurements to calculated (red lines) phase differences, using a single-resonance (dashed) model from Ref. [77] and its generalization to multiple resonances (solid). The calculations have been shifted by an overall phase offset to match the central value of the data. The data has been corrected for residual harmonic chirp using a krypton data set (see text). 
phase of the two-photon matrix element is given by

$$
\begin{aligned}
\Delta \phi(\omega)= & \operatorname{Arg}\left[\int d \Omega \tilde{E}_{X}(\Omega) \tilde{E}_{\omega}(\Omega-\omega)\right. \\
& \left.\times\left(\sum_{n} d_{n} \frac{\mathcal{E}_{n}+q_{n}}{\mathcal{E}_{n}+i}\right)\right],
\end{aligned}
$$

where $d_{n}$ is the amplitude for direct ionization, $\mathcal{E}_{n}=$ $2 / \Gamma_{n}^{R}\left(\hbar \Omega-E_{n}^{R}\right), E_{n}^{R}$ being the energy and $\Gamma_{n}^{R}$ the width of the resonant $(R)$ autoionizing state $n$, and $q_{n}$ is the Fano profile parameter for each autoionizing state. $\tilde{E}_{X}$ and $\tilde{E}_{\omega}$ are the Fourier transform of the APT and dressing IR field, respectively. In writing Eq. (5) we have also assumed that the variation of the phase of the dipole matrix element in the vicinity of $19.9 \mathrm{eV}$ is negligible. The resonance parameters in the range of 16.5-17.2 eV have been extracted from measurements by Chan et al. [78]. The absorption spectrum shows symmetric absorption features implying that ionization through the bound-state resonance dominates, leading to large $q$ parameters.

The dashed curve in Fig. 4 shows the model for a single dominant autoionizing state. The shape of this single-state curve is quite sensitive to the lifetime of the autoionizing resonance $(\Gamma)$, and somewhat insensitive to the Fano profile parameter $(q)$. Alternatively, using the four most prominent states to evaluate Eq. (5) leads to the solid curve. Surprisingly, this rather simple model matches extremely well with the measured data. The slight disagreement below $18.4 \mathrm{eV}$ is likely due to the underlying vibrational structure discussed above.

\section{METHODS}

Our attosecond pulse train and dressing IR field are produced by a titanium-doped:sapphire laser $(27 \mathrm{fs}, 30 \mathrm{~mJ}$, $100 \mathrm{~Hz}$ repetition rate) that is split into three copropagating beams: two temporally overlapped, high energy $(\sim 14 \mathrm{~mJ})$ beams used to drive high harmonic generation $(\mathrm{HHG})$ and produce the XUV APT, and a low energy $(\sim 1.5 \mathrm{~mJ})$ beam used as an interferometric probe. All three beams are spatially displaced and focused by a common $f=5 \mathrm{~m}$ focusing optic. Near the focus, the three beams spatially intersect in a 10-mm-long gas cell filled with $\sim 7$ torr of argon gas. A temporal advance $(\sim 150 \mathrm{fs})$ is introduced in the probe beam path so that it does not disrupt the HHG process when passing through the gas cell. The probe beam passes through a $100 \mu \mathrm{m}$ fused silica window to temporally overlap with the XUV APT. The crossed-beam geometry of the HHG-driving IR pulses separates the XUV and IR light in the far field (see SM Fig. S1) [74] so the residual drive laser light can be blocked downstream from the gas cell. The temporal delay of the weak IR field is controlled with a piezoelectric driven delay stage. Both the XUV APT and weak IR laser pulse are focused with a $\mathrm{B}_{4} \mathrm{C}$ coated focusing optic $(f=10 \mathrm{~cm})$ into the interaction region of a $1.2 \mathrm{~m}$ magnetic bottle spectrometer. The $\mathrm{B}_{4} \mathrm{C}$ coating's reflectance falls sharply near $31 \mathrm{eV}$, weakening the 19th harmonic and suppressing higher-energy photons. The $\mathrm{CO}_{2}$ target is introduced through a $35 \mu \mathrm{m}$ diameter gas needle near the interaction region. Electron spectra are recorded as a function of XUV/IR delay, resulting in modulation of the sideband features as seen in the bottom panel of Fig. 2. The phase offset, $\phi_{2 \omega}$ in Eq. (1), is recovered from the Fourier transform of the photoelectron spectrogram, which is averaged over the frequency range of the sideband oscillation.

The relevant term in Eq. (4) for theory comparison is the potential-dependent term, $\tau_{\mathrm{PI}}$. To extract this contribution from the measured phase offsets, we consider the phase differences between a signal $(s)$ and reference $(r)$ channel:

$$
\begin{aligned}
\Delta \tau^{(2)}\left(E_{2 n}\right) & =\frac{-1}{2 \omega}\left[\phi_{2 \omega}^{(s)}-\phi_{2 \omega}^{(r)}\right] \\
& =\Delta \tau_{\mathrm{PI}}\left(E_{2 n}\right)+\Delta \tau_{c c}\left(\epsilon, \epsilon^{\prime}\right),
\end{aligned}
$$

where $\epsilon=E_{2 n}-I_{p}^{(s)}$ and $\epsilon^{\prime}=E_{2 n}-I_{p}^{(r)}$ are the kinetic energies of the photoelectrons for the $2 n$th sideband peak in the signal and reference systems, $\Delta \tau_{c c}\left(\epsilon, \epsilon^{\prime}\right)$ is the difference in the continuum-continuum contribution due to the mismatch in photoelectron energies, and $\Delta \tau_{\mathrm{PI}}\left(E_{2 n}\right)$ is the differential photoionization delay we will compare with calculations. The spectral phase variation of the XUV pulse train, $\Delta \phi_{\mathrm{XUV}}$, has canceled out. When the relative difference between $\epsilon$ and $\epsilon^{\prime}$ is small, $\Delta \tau_{c c}\left(\epsilon, \epsilon^{\prime}\right)$ can be calculated very accurately [35], and subtracted from Eq. (6). For the measurements presented in Fig. 1(c) we reference the $B$-state photoionization delay to that of the $A$ state because the two channels have similar ionization potentials $\left(I_{p, A}=17.59 \mathrm{eV}\right.$ and $\left.I_{p, B}=18.07 \mathrm{eV}\right)$. The $X$-state channel $\left[I_{p, X}=13.77 \mathrm{eV}\right.$, Fig. $\left.1(\mathrm{~b})\right]$ is compared with a reference measurement made in krypton gas $\left(I_{p, \mathrm{Kr}}=\right.$ $14.00 \mathrm{eV})$. The krypton target is well studied [36] and its photoionization delay has been calculated [11], so we remove this contribution in the differential photoionization delay. $A$ and $B$-state values for $\Delta \tau^{(2)}\left(E_{2 n}\right)$ referenced with krypton are made available in the Supplemental Material [74].

$\mathrm{XUV} / \mathrm{IR}$ delay scans were performed for the target, $\mathrm{CO}_{2}$, and reference, $\mathrm{Kr}$, in alternate fashion. The data presented in Fig. 1 is the result of 51 independent measurement scans. Comparing the phase offset across the 51 independent measurement scans requires precise knowledge of any drifts in the XUV/IR laser delay. We use the interleaved measurements of the krypton reference to track the long-term drift of the delay stage. For each $\mathrm{Kr}$ scan, the extracted sideband offset from spectral integration is combined into a weighted average,

$$
\Phi=\operatorname{Arg}\left[\sum_{n=S B} A_{2 \omega}\left(E_{n}\right) e^{i \cdot \phi_{2 \omega}\left(E_{n}\right)}\right],
$$

which is used to track the delay-stage drift. A smoothing spline is fit to the experimental values of $\Phi$ and is used to interpolate the value of $\Phi$ at each $\mathrm{CO}_{2}$ scan. We subtract the interpolated value of $\Phi$ from each $\mathrm{CO}_{2}$ scan to account for delaystage drift. This procedure accounts for most but not all of the delay-stage artifact. There is a residual artifact between two individual scans due to uncompensated drift between experiments. Each experiment reproduces similar trend lines across the sampled photoelectron energies; however, the individual experiments still exhibit a systematic phase shift. For each $\mathrm{CO}_{2}$ data set we again calculate the weighted sideband offset. Removing this weighted offset yields the final results shown in Fig. 1. We interpret this behavior as a systematic uncertainty 
in the zero-phase measurement, which we remove, along with a smaller random variation in the measured phase offset.

The calculations presented in Fig. 1 show two-photon $\mathrm{XUV}+\mathrm{IR}$ photoionization time delays for the independent channel and coupled-channel models. The single photon photoionization probability amplitude, $c_{n}(\vec{k})$, for weak XUV pulses can be calculated from first-order perturbation theory:

$$
\begin{gathered}
c_{n}(\vec{k})=\tilde{E}_{\mathrm{X}}\left(\omega=\frac{k^{2}+2 I_{p, n}}{2 \hbar}\right) \cdot d_{n}(\vec{k}), \\
d_{n}(\vec{k})=\left\langle\psi_{\vec{k}, n}^{(-)}|\hat{\mu} \cdot \vec{r}| \Psi_{0}\right\rangle,
\end{gathered}
$$

where $d_{n}(\vec{k})$ is the dipole matrix element between the outgoing $\left(\psi_{\vec{k}, n}^{(-)}\right)$and ground-state $\left(\Psi_{0}\right)$ wave functions in the length gauge and $\hat{\mu}$ is the polarization direction in the molecular frame. The channel-resolved dipole matrix element, Eq. (9), is computed using the complex Kohn method for photoionization [38-46,54-63]. The calculations use explicit representations of the initial neutral state and of the final cationic states obtained with one single 11-orbital basis. This basis for the neutral and cationic states was obtained using a state-averaged multiconfiguration self-consistent-field (MCSCF) calculation performed with the COLUMBUS quantum chemistry program [79-83]. The orbitals obtained are a compromise between those optimized for the neutral and cationic states. The primitive basis for this MCSCF calculation was Dunning's aug$c c-p V D Z$ basis set [84], with additional basis functions on the oxygen atom as described in the Supplemental Material [74]. The outgoing wave function, $\psi_{n}^{(-)}$, is expanded in a partial-wave representation $(l, m)$, up to $l=3$, and the dipole matrix element is calculated for transitions between these functions and the initial ground state within the approximation of separable exchange [38,39,43].

All meaningful partial waves are then coupled by the IR dressing field, as shown in the Appendix, and described in Ref. [7], to determine the molecular frame (MF) two-photon photoionization time delays. These MF photoionization time delays are then averaged over both the polarization direction and outgoing electron direction to approximate the experimental conditions. This averaged quantity is what we refer to as the laboratory-frame (LF) photoionization delay as defined in Eq. (4) and plotted in Fig. 1. The single photon LF photoionization delays and two-photon MF photoionization delays for all accessible final state channels are shown in the Supplemental Material [74].

\section{CONCLUSIONS}

These measurements and calculations demonstrate effects of electron correlation on time delays in photoionization of molecules. Photoionization time delays are a direct and easy-to-understand manifestation of the effective interaction that an outgoing electron experiences. We have observed two effects of electron correlation in time-domain measurements of $\mathrm{CO}_{2}$ photoionization, via (1) a shape resonance and (2) autoionizing states, demonstrating that the inclusion of electronic correlation is important when considering resonant features in molecular photoionization with XUV light. While this seems clear for autoionizing states, which are inherently multichannel phenomena, this result is somewhat surprising for shape resonance features which are typically considered to be single channel phenomena. For a shape resonance, we have shown that electron-electron interactions provide dynamic modifications to the effective potential that can be directly observed in all final state channels. These results highlight the need for including electron correlation when describing time-domain measurements of photoionization of molecular targets, where autoionizing states and shape resonances are omnipresent.

Molecular control (e.g., molecular alignment) techniques can be applied to isolate specific cationic states of $\mathrm{CO}_{2}{ }^{+}$ in future experiments. Continuum-resolved molecular frame measurements will further elucidate the dependence of electron correlation on molecular orientation and electron emission angle. These experiments could be further improved by varying the XUV frequency comb spacing, thus mapping out more energy points in the differential scattering phase.

\section{ACKNOWLEDGMENTS}

This work was supported by the AMOS program within the Chemical Sciences, Geosciences, and Biosciences Division of the Office of Basic Energy Sciences, Office of Science, U.S. Department of Energy. Raw and analyzed data, both experimental and theoretical, along with a subset of the analysis code is available at [85].

\section{APPENDIX: TWO-PHOTON PHOTOIONIZATION DELAYS}

\section{Channel coupling in two-color photoionization}

The interferometric ionization technique used in this work measures the delay in two-color photoionization defined as [7,35]

$$
\tau_{n}^{(2)}(2 q, \hat{k}, \hat{R})=\frac{1}{2 \omega} \operatorname{Arg}\left[M_{n}^{(2) *}\left(\vec{k} ; \epsilon_{0}+\hbar \Omega_{2 q+1}, \hat{R}\right) M_{n}^{(2)}\left(\vec{k} ; \epsilon_{0}+\hbar \Omega_{2 q-1}, \hat{R}\right)\right],
$$

where $\vec{k}$ is the final momentum of the outgoing electron for ionic channel $n, \hat{R}$ defines the orientation of the molecular axis relative to the laser polarization, and $\hbar \Omega_{2 q \pm 1}$ are the energies of the IR-coupled, XUV photons, which interfere to create the $2 q$ th sideband. We assume that $\hbar \Omega_{2 q \pm 1}$ is larger than any of the relevant channel binding energies. The two-photon ionization matrix element $\left(M_{n}^{(2)}\right)$ for channel $n$ is given by

$$
M_{n}^{(2)}\left(\vec{k} ; \epsilon_{0}+\Omega, \hat{R}\right)=-i \sum_{p} \int d \epsilon_{i} \frac{\left\langle\Psi_{\vec{k}, n}^{(-)}\left|\vec{E}_{\omega} \cdot \vec{r}\right| \Psi_{i, p}\right\rangle\left\langle\Psi_{i, p}\left|\vec{E}_{\mathrm{X}} \cdot \vec{r}\right| \Psi_{0}\right\rangle}{\epsilon_{0}+\hbar \Omega-\epsilon_{i}},
$$


where $\Psi_{\vec{k}, n}^{(-)}$is the observed final state wave function of momentum $\vec{k}$ in ionic channel $n$ and $\Psi_{i, p}$ is the intermediate state of energy $\epsilon_{i}$ in ionic channel $p$ produced by XUV ionization of the ground state, $\Psi_{0}$ with negative energy $\epsilon_{0}$. In Eq. (A2) we have ignored the far off-resonance matrix element, where the IR pulse interacts before the XUV pulse. Thus Eq. (A1) is both emission angle and orientation resolved. Recently, Douguet $e t$ al. developed a full numerical theory to describe $M_{n}^{(2)}$ using the complex Kohn variational method [86]. Here we develop an intermediate theory, similar to that originally developed by Dahlstrom et al. [1], which focuses on the long-range behavior of the wave function. For simplicity, we assume that both the XUV and IR fields are copolarized and linear. Our approach for analyzing Eq. (A2) is to express the scattering wave functions using a close-coupling expansion

$$
\left|\Psi_{\vec{k}, n}^{(-)}\right\rangle=\sum_{p} \int d^{3} \vec{r} F_{p, n \vec{k}}(\vec{r}) a^{\dagger}(\vec{r})\left|\Phi_{p}\right\rangle
$$

in which $\Phi_{p}$ are the $(N-1)$-electron wave functions for the residual ion in channel $p, a^{\dagger}(\vec{r})$ is the creation operator for a (properly spin-coupled) electron at position $\vec{r}$, and $F_{p, n \vec{k}}$ is a single electron wave function. The sum over cation channels $p$ in Eq. (A3) is complete, but the asymptotic form of the wave function concerns only the channels $p, n$ for which photoionization is allowed. The asymptotic form of the single-electron wave functions $F_{p, n}$ for open channels is encoded by the $S$ matrix,

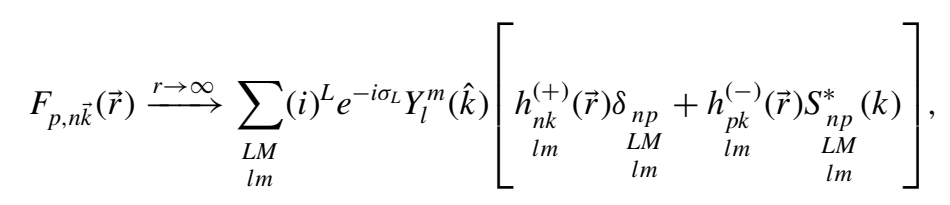

where $Y_{L}^{M}$ are the spherical harmonic functions, $\sigma_{L}=\arg [\Gamma(L+1-i / k)]$ is the scattering phase shift induced by the Coulomb potential, and $\Gamma$ is the complex gamma function. We have defined the regular-incoming $\left(h^{(+)}\right)$and irregular-outgoing $\left(h^{(-)}\right)$ Coulomb waves as

$$
\begin{aligned}
& h_{\substack{n k \\
L M}}^{(+)}=\frac{1}{\sqrt{2}}\left(\begin{array}{c}
f_{n k}+i g_{n k} \\
L M
\end{array}\right) \text {, } \\
& h_{n k}^{(-)}=\frac{1}{\sqrt{2}}\left(\begin{array}{c}
f_{n k}-i g_{n k} \\
L M
\end{array}\right) \text {, } \\
& \underset{L M}{f_{n k}} \stackrel{r \rightarrow \infty}{\longrightarrow} \frac{N_{k}}{r} \sin \left[k r+\Theta_{k L}(r)\right] Y_{L}^{M}(\hat{r}), \\
& \underset{L M}{g_{n k}} \stackrel{r \rightarrow \infty}{\longrightarrow} \frac{N_{k}}{r} \cos \left[k r+\Theta_{k L}(r)\right] Y_{L}^{M}(\hat{r}) .
\end{aligned}
$$

$f$ and $g$ are the regular and irregular Coulomb function, respectively, and $N_{k}=\sqrt{2 / \pi k}$ is a normalization constant. The phase of the Coulomb functions is given by

$$
\Theta_{k L}(r)=\frac{1}{k} \ln (2 k r)-\frac{L \pi}{2}+\sigma_{L} .
$$

Using Eqs. (A3) and (A4) we can rewrite the matrix element between the intermediate and final states, $\left\langle\Psi_{\vec{k}, n}^{(-)}|\vec{r}| \Psi_{i, p}\right\rangle$,

$$
\begin{aligned}
\left\langle\Psi_{\vec{k}, n}^{(-)}|\vec{r}| \Psi_{i, p}\right\rangle & =\sum_{q q^{\prime}}\left\langle\Phi_{q} F_{q, n k}|\vec{r}| \Phi_{q^{\prime}} F_{q^{\prime}, p \kappa}\right\rangle \\
& =\sum_{q q^{\prime}}\left\langle\Phi_{q}|\vec{r}| \Phi_{q^{\prime}}\right\rangle\left\langle F_{q, n k} \mid F_{q^{\prime}, p \kappa}\right\rangle+\delta_{q q^{\prime}}\left\langle F_{q, n k}|\vec{r}| F_{q^{\prime}, p \kappa}\right\rangle \\
& =\sum_{q}\left\langle F_{q, n k}|\vec{r}| F_{q, p \kappa}\right\rangle,
\end{aligned}
$$

where we have assumed that there are no resonant transitions between the residual ionic states. Following the approach laid out by Dahlstrom et al. we rewrite Eq. (A2) using Eq. (A3),

$$
M_{n}^{(2)}\left(\vec{k} ; \epsilon_{0}+\Omega, \hat{R}\right)=\frac{1}{i} \sqrt{\frac{4 \pi}{3}} E_{\omega} E_{X} \sum_{\mu \mu^{\prime}} D_{0 \mu}^{(1)}(\hat{R}) D_{0 \mu^{\prime}}^{(1)}(\hat{R}) \sum_{q}\left\langle F_{q, n k}\left|r Y_{1}^{\mu^{\prime}}(\hat{r})\right| \rho_{q \mu, \kappa}\right\rangle,
$$


where $D_{l k}^{(j)}(\hat{R})$ is the Wigner matrix which rotates the polarization of the XUV and IR pulses into the molecular frame, and we have defined the first-order perturbed wave function for the $q$ th channel, $\rho_{q \mu, \kappa}(r)$, as

$$
\rho_{q \mu, \kappa}=\sum_{p} \int d \epsilon_{\kappa} d \hat{\Omega}_{\hat{\kappa}} \frac{F_{q, p \kappa}(\vec{r})\left\langle\psi_{\vec{\kappa}, p}^{(-)}\left|r_{\mu}\right| \Phi_{0}\right\rangle}{\epsilon_{0}+\hbar \Omega-\epsilon_{\kappa}},
$$

where $r_{\mu}=r Y_{1}^{\mu}(\hat{r})$. Using Eq. (A4) as an equality we rewrite $\rho_{q, \kappa}$,

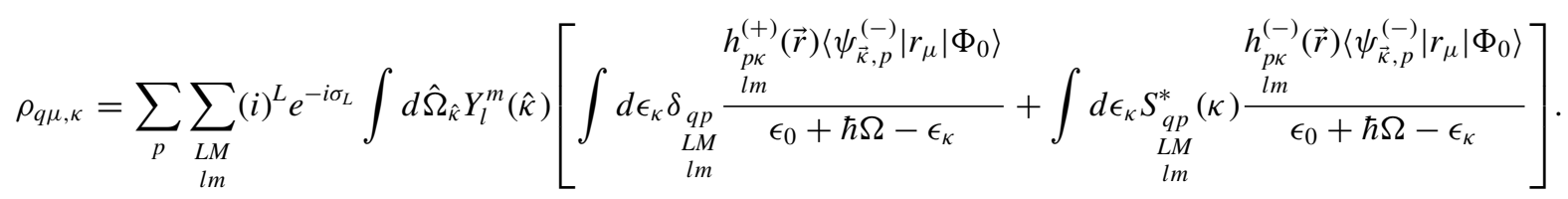

We rewrite the asymptotic form on Eq. (B9) using the following relations from Ref. [35]:

$$
\begin{aligned}
\int d \epsilon_{\kappa} \frac{f_{p \kappa}\left\langle\psi_{\vec{\kappa}, p}^{(-)}\left|r_{\mu}\right| \Phi_{0}\right\rangle}{\epsilon_{0}+\hbar \Omega-\epsilon_{\kappa}} & =-\frac{\pi N_{\kappa}}{r} Y_{L}^{M}(\hat{r}) \exp \left[i \kappa r+i \Theta_{\kappa L}(r)\right]\left\langle\psi_{\vec{\kappa}, p}^{(-)}\left|r_{\mu}\right| \Phi_{0}\right\rangle, \\
\int d \epsilon_{\kappa} \frac{L M}{g_{p \kappa}\left\langle\psi_{\vec{\kappa}, p}^{(-)}\left|r_{\mu}\right| \Phi_{0}\right\rangle} & =-i \frac{\pi N_{\kappa}}{r} Y_{L}^{M}(\hat{r}) \exp \left[i \kappa r+i \Theta_{\kappa L}(r)\right]\left\langle\psi_{\vec{\kappa}, p}^{(-)}\left|r_{\mu}\right| \Phi_{0}\right\rangle .
\end{aligned}
$$

Then $\rho_{q, \kappa}$ becomes

$$
\rho_{q \mu, \kappa}=-\frac{\pi N_{\kappa}}{r} \sum_{p} \sum_{\substack{L M \\ l m}} Y_{l}^{m}(\hat{r}) S_{\substack{L q \\ l m}}^{*}(\kappa) \exp \left[i \kappa r+\frac{i}{\kappa} \ln (2 \kappa r)\right] I_{p, l m \mu}(\kappa)
$$

where

$$
I_{p, l m \mu}(\kappa)=(i)^{l} e^{-i \sigma_{l}(\kappa)} \int d \hat{\Omega}_{\hat{\kappa}} Y_{l}^{m}(\hat{\kappa})\left\langle\psi_{\vec{\kappa}, p}^{(-)}\left|r_{\mu}\right| \Phi_{0}\right\rangle
$$

is the partial wave expansion of the XUV dipole matrix element for ionization to the $p$ channel [Eq. (9)], which is calculated using the complex Kohn approach, as described in the main text.

Next we must evaluate the matrix element, $\left\langle F_{q, n k}\left|r Y_{1}^{\mu^{\prime}}\right| \rho_{q \mu, \kappa}\right\rangle$,

$$
\begin{aligned}
& \left\langle F_{q, n k}\left|r Y_{1}^{\mu^{\prime}}\right| \rho_{q \mu, \kappa}\right\rangle=-\pi N_{k} N_{\kappa} \sum_{p} \sum_{\substack{L M \\
L^{\prime} M^{\prime}}}(-i)^{L} e^{i \sigma_{L}(k)} \sum_{\substack{l m \\
l^{\prime} m^{\prime}}} Y_{l}^{m}(\hat{k})\left\langle Y_{l}^{m}\left|Y_{1}^{\mu^{\prime}}\right| Y_{l^{\prime}}^{m^{\prime}}\right| I_{p, l^{\prime} m^{\prime} \mu}(\kappa)
\end{aligned}
$$

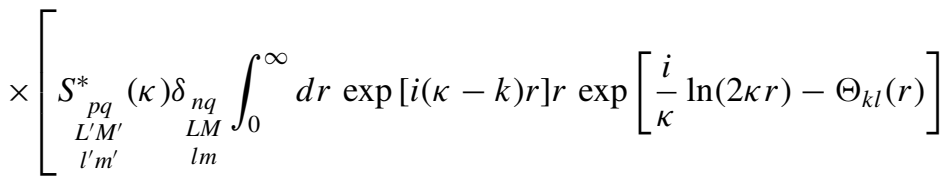

$$
\begin{aligned}
& \left.+\underset{\substack{p q \\
L^{\prime} M^{\prime} \\
l^{\prime} m^{\prime}}}{* \underset{L M}{l m}}(\kappa) S_{\substack{q n \\
l m}}(k) \int_{0}^{\infty} d r \exp [i(\kappa+k) r] r \exp \left[\frac{i}{\kappa} \ln (2 \kappa r)+\Theta_{k l}(r)\right]\right] .
\end{aligned}
$$

We define the following integrals:

$$
J_{ \pm}(\kappa, k)= \pm \frac{1}{2 i} \int_{0}^{\infty} d r r^{1+i(1 / \kappa \pm 1 / k)} \exp [i(\kappa \pm k) r]
$$

and rewrite Eq. (A12),

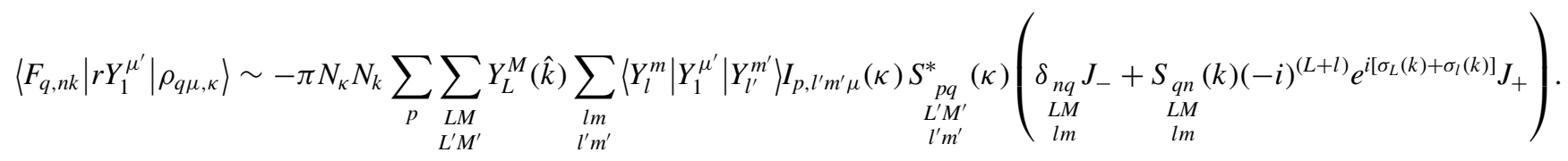


As described in Ref. [35], the contribution from the $J_{+}$integral is much smaller than that of the $J_{-}$integral. This is because the IR photon energy is small compared to the final electron energy, and thus $k^{2} / 2-\kappa^{2} / 2=\omega<k^{2} / 2$. Therefore, the difference $|\kappa-k|$ is much smaller than the sum $\kappa+k$ and the fast oscillations of $\exp [i(\kappa+k) r]$ in the $J_{+}$integral lead to a cancellation. Thus we arrive at the expression,

$$
\left\langle F_{q, n k}\left|r Y_{1}^{\mu^{\prime}}\right| \rho_{q \mu, \kappa}\right\rangle=-\pi N_{k} N_{\kappa} \sum_{p} \sum_{\substack{L M \\ L^{\prime} M^{\prime} \\ l^{\prime} m^{\prime}}} Y_{L}^{M}(\hat{k}) I_{p, l^{\prime} m^{\prime} \mu}(\kappa)\left\langle Y_{L}^{M}\left|Y_{1}^{\mu^{\prime}}\right| Y_{l^{\prime}}^{m^{\prime}}\right| S_{\substack{p q \\ L^{\prime} M^{\prime} \\ l^{\prime} m^{\prime}}}^{*}(\kappa) \delta_{n q} J_{-}(\kappa, k)
$$

Using Eq. (A13) we arrive at the following simplification for Eq. (A2):

$$
M_{n}^{(2)}\left(\vec{k} ; \epsilon_{0}+\Omega, \hat{R}\right)=\frac{4 \pi^{2}}{3 i} E_{\omega} E_{X} \sum_{\mu \mu^{\prime}} D_{0 \mu}^{(1)}(\hat{R}) D_{0 \mu^{\prime}}^{(1)}(\hat{R}) N_{k} N_{\kappa} \sum_{p} \sum_{\substack{L M \\ L^{\prime} M^{\prime}}} Y_{L}^{M}(\hat{k}) \sum_{l^{\prime} m^{\prime}} I_{p, l^{\prime} m^{\prime} \mu}(\kappa)\left\langle Y_{L}^{M}\left|Y_{1}^{\mu^{\prime}}\right| Y_{l^{\prime}}^{m^{\prime}}\right| S_{\substack{p n \\ L^{\prime} M^{\prime} \\ l^{\prime} m^{\prime}}}(\kappa) J_{-}(\kappa, k)
$$

This can be recast in the same form as the expression derived by Baykusheva and Wörner [7]:

$$
M_{n}^{(2)}\left(\vec{k} ; \epsilon_{0}+\Omega, \hat{R}\right)=N_{k} N_{\kappa} J_{-}(k, \kappa) \sum_{L M} b_{n, L M}(\kappa ; \hat{R}) Y_{L}^{M}(\hat{k})
$$

But now $b_{n, L M}$ is defined as

$$
b_{n, L M}(\kappa ; \hat{R})=\frac{4 \pi}{3 i} E_{\omega} E_{X} \sum_{\mu \mu^{\prime}} D_{0 \mu}^{(1)}(\hat{R}) D_{0 \mu^{\prime}}^{(1)}(\hat{R}) \sum_{p} \sum_{\substack{L^{\prime} M^{\prime} \\ l^{\prime} m^{\prime}}} I_{p, l^{\prime} m^{\prime} \mu}(\kappa)\left\langle Y_{L}^{M}\left|Y_{1}^{\mu^{\prime}}\right| Y_{l^{\prime}}^{m^{\prime}}\right\rangle S_{\substack{p n \\ L^{\prime} M^{\prime} \\ l^{\prime} m^{\prime}}}^{*}(\kappa)
$$

The angle- and orientation-dependent delay in Eq. (A1) is then given by

$$
\begin{aligned}
\tau_{n}^{(2)}(2 q, \hat{k}, \hat{R}) & =\frac{1}{2 \omega}\left\{\operatorname{Arg}\left[N_{k-\omega} J_{-}(k, k-\omega) N_{k+\omega}^{*} J_{-}^{*}(k, k+\omega)\right]+\operatorname{Arg}\left[\sum_{\left.\left.\begin{array}{c}
L M \\
L^{\prime} M^{\prime}
\end{array} Y_{L}^{M *}(\hat{k}) Y_{L^{\prime}}^{M^{\prime}}(\hat{k}) b_{n, L M}^{*}(k-\omega ; \hat{R}) b_{n, L^{\prime} M^{\prime}}(k+\omega ; \hat{R})\right]\right\}}\right]\right)=\tau_{c c}(k)+\tau_{P I}(2 q, \hat{k}, \hat{R}) .
\end{aligned}
$$

As pointed out in Ref. [7], the first term in Eq. (A18) only depends on the photon energy and can be interpreted as a continuumcontinuum delay $\left(\tau_{c c}\right)$. The second term in Eq. (A18) is again a target-specific delay ( $\left.\tau_{P I}\right)$, but the single photon delays are modified by the interaction with the IR light. In addition to this modification, described by Baykusheva and Wörner, Eq. (A17) shows that the channel coupling adds additional modifications. In the absence of any channel coupling, the $S$ matrix is purely diagonal; Eq. (A17) reduces to the expression derived by Baykusheva and Wörner.

\section{Molecular photoionization delays}

The partial wave decomposition of the single-photon dipole transition matrix elements are calculated as described at the end of the main text. The results are used to calculate $b_{L M}(k ; \hat{R})$ described in Eq. (A17). For the calculation presented in the main text, we make the assumption that the $S$ matrix in Eq. (A17) is purely diagonal. In this approximation, we fully include channel coupling in the single-photon ionization process, but neglect the effect of the channel coupling in the interaction with the IR photon. The result is the emission angle $(\hat{k})$ - and orientation angle $(\hat{R})$-resolved photoionization delays for a central photon energy of $\hbar \Omega_{2 q}$. The calculated molecular frame (MF) photoionization delays are shown in the Supplemental Material.

\section{Angular averaging}

There are a number of interesting features in the MF, angle-resolved photoemission delays; however, our experiments use a randomly oriented target gas and magnetic bottle electron time-of-flight spectrometer which does not resolve emission angle. Therefore, Eq. (A18) needs to be averaged over both the molecular axis distribution or the outgoing electron angle. The contributions from each pair of $(\hat{k}, \hat{R})$ are incoherently averaged, weighed by a cross section term $\mid b_{n, L M}^{*}(k-\omega ; \hat{R}) b_{n, L^{\prime} M^{\prime}}(k+$ 
$\omega ; \hat{R}) \mid$. The measured photoionization time delays are then compared to the calculated average,

$$
\begin{aligned}
\tau_{\mathrm{PI}}(2 q) & =\frac{1}{2 \omega} \operatorname{Arg}\left[\int d \hat{\Omega}_{\hat{R}} \int d \hat{\Omega}_{\hat{k}} \sum_{\substack{L M \\
L^{\prime} M^{\prime}}} Y_{L}^{M *}(\hat{k}) Y_{L^{\prime}}^{M^{\prime}}(\hat{k}) b_{n, L M}^{*}(k-\omega ; \hat{R}) b_{n, L^{\prime} M^{\prime}}(k+\omega ; \hat{R})\right] \\
& =\frac{1}{2 \omega} \operatorname{Arg}\left[\sum_{L M} \int d \hat{\Omega}_{\hat{R}} b_{n, L M}^{*}(k-\omega ; \hat{R}) b_{n, L M}(k+\omega ; \hat{R})\right]
\end{aligned}
$$

in Fig. 1 of the main text.

\section{Photoionization delays in the presence of an intermediate bound-state resonance}

Recently, it was shown by Argenti et al. that, when intermediate bound-state resonances occur in the two-photon ionization process, the two-photon photoionization delay $\left(\tau^{(2)}\right)$ no longer has a scattering counterpart [4]. This is a result of the formation of a new pathway from the resonant bound state to the continuum through radiative coupling (i.e., direct ionization of the bound state). The theory developed in Sec. 1 neglects this radiative pathway. This assumption is validated by the quality of the agreement between the model and our data in Fig. 4. Similar agreement was observed in the recent article by Barreau et al. between a model neglecting radiative coupling, TDSE simulation, and measured data [25]. In general, radiative coupling of the bound state to the continuum should be included in any model of resonant photoionization, but the agreement shown in Fig. 4 leads to the conclusion that this coupling is quite small in this instance.

In $\mathrm{CO}_{2}$ the autoionizing states form a Rydberg series converging to the $A$ - and $B$-state continuum. This means the resonant states are dominated by a configuration where an electron from either HOMO-1 or HOMO-2 single electron orbital has been promoted to an unoccupied Rydberg orbital. Electron correlation couples these states to the $X$-state continuum which is dominated by an electron configuration where an electron is removed from the HOMO. The coupling between the autoionizing and continuum states is quite strong, as the intermediate autoionizing states have lifetimes on the order of $10 \mathrm{fs}$. The radiative coupling between the autoionizing intermediate states and the $X$-state continuum states is comparatively weaker because the transition requires the rearrangement of two electrons, and the dipole operator typically acts on a single electron. There is likely to be strong radiative coupling of the intermediate autoionizing state to either the $A$ - or $B$-state continuum. However, this coupling would produce a very low-energy electron $(<1 \mathrm{eV})$ in the $A$ - or $B$-state continuum. In Fig. 4 we analyze the effect of the autoionizing states in the vicinity of the electron kinetic-energy spectrum near $4 \mathrm{eV}$, which is therefore unaffected by the radiative coupling to another continuum.

We can extend this discussion to include the molecular shape resonances. In the case of the shape resonance, the coupling between the trapped portion of the wave function and the continuum is very strong; the resonance decays within 100 as. Such a coupling is sure to dominate over any dipole coupling via the weak IR field.

\section{Model for autoionization}

Starting from Eq. (A16), we can derive the simplified model given in Eq. (5). Following Fano's original formulation we start with an unperturbed Hamiltonian which supports a bound state $|R\rangle$ and a featureless continuum $|\epsilon\rangle$. The "configuration interaction" couples only the bound state to the continuum. In this formalism the dipole transition matrix element between the initial state and eigenstate of the full Hamiltonian can be written as

$$
\left\langle\psi_{E}^{(-)}\left|r_{\mu}\right| \Phi_{0}\right\rangle=d_{\epsilon} \frac{\mathcal{E}+q}{\mathcal{E}-i}, \quad d_{\epsilon}=\left\langle\epsilon\left|r_{\mu}\right| \Phi_{0}\right\rangle,
$$

where $i$ is the imaginary unit and $\left|\psi_{E}^{(-)}\right\rangle$is the Fano solution [77,87]. The reduced energy $\mathcal{E}$ and the Fano parameter $q$ are defined in the main text after Eq. (5). Furthermore, using the $|\epsilon\rangle$ channel as a reference, the $S$-scattering matrix is given by [77]

$$
S^{*}(\kappa)=\frac{(\mathcal{E}-i)}{(\mathcal{E}+i)},
$$

where $\kappa=\sqrt{2\left(\hbar \Omega-I_{p}\right)}$. Substituting these expressions into Eq. (A17), and neglecting the angular components,

$$
b(\kappa)=d_{\kappa} \frac{\mathcal{E}+q}{\mathcal{E}-i} \frac{\mathcal{E}-i}{\mathcal{E}+i}=d_{\epsilon} \frac{\mathcal{E}+q}{\mathcal{E}+i} .
$$

This is precisely the same expression derived by Jiménez-Galán et al. assuming that the bound state $|r\rangle$ is not radiatively coupled to the continuum. This is the same approximation made in deriving Eq. (A16). 
[1] J. M. Dahlström, A. L'Huillier, and A. Maquet, Introduction to attosecond delays in photoionization, J. Phys. B: At., Mol., Opt. Phys. 45, 183001 (2012).

[2] K. Klünder, J. M. Dahlström, M. Gisselbrecht, T. Fordell, M. Swoboda, D. Guénot, P. Johnsson, J. Caillat, J. Mauritsson, A. Maquet, R. Taïeb, and A. L'Huillier, Probing Single-Photon Ionization on the Attosecond Time Scale, Phys. Rev. Lett. 106, 143002 (2011).

[3] V. V. Serov, V. L. Derbov, and T. A. Sergeeva, Interpretation of the time delay in the ionization of Coulomb and two-center systems, Phys. Rev. A 87, 063414 (2013).

[4] L. Argenti, Á. Jiménez-Galán, J. Caillat, R. Taïeb, A. Maquet, and F. Martín, Control of photoemission delay in resonant twophoton transitions, Phys. Rev. A 95, 043426 (2017).

[5] R. Pazourek, S. Nagele, and J. Burgdörfer, Attosecond chronoscopy of photoemission, Rev. Mod. Phys. 87, 765 (2015).

[6] M. Ossiander, F. Siegrist, V. Shirvanyan, R. Pazourek, A. Sommer, T. Latka, A. Guggenmos, S. Nagele, J. Feist, J. Burgdörfer, R. Kienberger, and M. Schultze, Attosecond correlation dynamics, Nat. Phys. 13, 280 (2017).

[7] D. Baykusheva and H. J. Wörner, Theory of attosecond delays in molecular photoionization, J. Chem. Phys. 146, 124306 (2017).

[8] M. Schultze, M. Fieß, N. Karpowicz, J. Gagnon, M. Korbman, M. Hofstetter, S. Neppl, A. L. Cavalieri, Y. Komninos, Th. Mercouris, C. A. Nicolaides, R. Pazourek, S. Nagele, J. Feist, J. Burgdörfer, A. M. Azzeer, R. Ernstorfer, R. Kienberger, U. Kleineberg, E. Goulielmakis, F. Krausz, and V. S. Yakovlev, Delay in photoemission, Science 328, 1658 (2010).

[9] D. Guénot, K. Klünder, C. L. Arnold, D. Kroon, J. M. Dahlström, M. Miranda, T. Fordell, M. Gisselbrecht, P. Johnsson, J. Mauritsson, E. Lindroth, A. Maquet, R. Taïeb, A. L'Huillier, and A. S. Kheifets, Photoemission-time-delay measurements and calculations close to the $3 s$-ionization-crosssection minimum in Ar, Phys. Rev. A 85, 053424 (2012).

[10] A. S. Kheifets, Time delay in valence-shell photoionization of noble-gas atoms, Phys. Rev. A 87, 063404 (2013).

[11] A. Kheifets, A. Mandal, P. C. Deshmukh, V. K. Dolmatov, D. A. Keating, and S. T. Manson, Relativistic calculations of angledependent photoemission time delay, Phys. Rev. A 94, 013423 (2016).

[12] D. Guénot, D. Kroon, E. Balogh, E. W. Larsen, M. Kotur, M. Miranda, T. Fordell, P. Johnsson, J. Mauritsson, M Gisselbrecht, K. Varjù, C. L. Arnold, T. Carette, A. S. Kheifets, E. Lindroth, A. L'Huillier, and J. M. Dahlström, Measurements of relative photoemission time delays in noble gas atoms, J. Phys. B: At., Mol., Opt. Phys. 47, 245602 (2014).

[13] C. Palatchi, J. M. Dahlström, A. S. Kheifets, I. A. Ivanov, D. M. Canaday, P. Agostini, and L. F. DiMauro, Atomic delay in helium, neon, argon and krypton, J. Phys. B: At., Mol., Opt. Phys. 47, 245003 (2014).

[14] M. Sabbar, S. Heuser, R. Boge, M. Lucchini, T. Carette, E. Lindroth, L. Gallmann, C. Cirelli, and U. Keller, Resonance Effects in Photoemission Time Delays, Phys. Rev. Lett. 115, 133001 (2015).

[15] M. Isinger, R. J. Squibb, D. Busto, S. Zhong, A. Harth, D. Kroon, S. Nandi, C. L. Arnold, M. Miranda, J. M. Dahlström, E. Lindroth, R. Feifel, M. Gisselbrecht, and A. L'Huillier,
Photoionization in the time and frequency domain, Science 358, 893 (2017).

[16] D. Kiesewetter, R. R. Jones, A. Camper, S. B. Schoun, P. Agostini, and L. F. DiMauro, Probing electronic binding potentials with attosecond photoelectron wavepackets, Nat. Phys. 14, 68 (2017).

[17] S. Haessler, B. Fabre, J. Higuet, J. Caillat, T. Ruchon, P. Breger, B. Carré, E. Constant, A. Maquet, E. Mével, P. Salières, R. Taïeb, and Y. Mairesse, Phase-resolved attosecond nearthreshold photoionization of molecular nitrogen, Phys. Rev. A 80, 011404(R) (2009).

[18] M. Huppert, I. Jordan, D. Baykusheva, A. von Conta, and H. J. Wörner, Attosecond Delays in Molecular Photoionization, Phys. Rev. Lett. 117, 093001 (2016).

[19] J. Vos, L. Cattaneo, S. Patchkovskii, T. Zimmermann, C. Cirelli, M. Lucchini, A. Kheifets, A. S. Landsman, and U. Keller, Orientation-dependent stereo Wigner time delay and electron localization in a small molecule, Science 360, 1326 (2018).

[20] L. Cattaneo, J. Vos, R. Y. Bello, A. Palacios, S. Heuser, L. Pedrelli, M. Lucchini, C. Cirelli, F. Martín, and U. Keller, Attosecond coupled electron and nuclear dynamics in dissociative ionization of $\mathrm{H}_{2}$, Nat. Phys. 14, 733 (2018).

[21] V. Gruson, L. Barreau, Á Jiménez-Galan, F. Risoud, J. Caillat, A. Maquet, B. Carré, F. Lepetit, J.-F. Hergott, T. Ruchon, L. Argenti, R. Taïeb, F. Martín, and P. Salières, Attosecond dynamics through a Fano resonance: Monitoring the birth of a photoelectron, Science 354, 734 (2016).

[22] M. Kotur, D. Guénot, Á Jiménez-Galán, D. Kroon, E. W. Larsen, M. Louisy, S. Bengtsson, M. Miranda, J. Mauritsson, C. L. Arnold, S. E. Canton, M. Gisselbrecht, T. Carette, J. M. Dahlström, E. Lindroth, A. Maquet, L. Argenti, F. Martín, and A. L'Huillier, Spectral phase measurement of a Fano resonance using tunable attosecond pulses, Nat. Commun. 7, 10566 (2016).

[23] C. Cirelli, C. Marante, S. Heuser, C. L. M. Petersson, Á. Jiménez Galán, L. Argenti, S. Zhong, D. Busto, M. Isinger, S. Nandi, S. Maclot, L. Rading, P. Johnsson, M. Gisselbrecht, M. Lucchini, L. Gallmann, J. M. Dahlström, E. Lindroth, A. L'Huillier, F. Martín, and U. Keller, Anisotropic photoemission time delays close to a Fano resonance, Nat. Commun. 9, 955 (2018).

[24] D. Busto, L. Barreau, M. Isinger, M. Turconi, C. Alexandridi, A. Harth, S. Zhong, R. J. Squibb, D. Kroon, S. Plogmaker, M Miranda, Á Jiménez-Galán, L. Argenti, C. L. Arnold, R. Feifel, F. Martín, M. Gisselbrecht, A. L'Huillier, and P. Salières, Timefrequency representation of autoionization dynamics in helium, J. Phys. B: At., Mol., Opt. Phys. 51, 044002 (2018).

[25] L. Barreau, C. L. M. Petersson, M. Klinker, A. Camper, C. Marante, T. Gorman, D. Kiesewetter, L. Argenti, P. Agostini, J. González-Vázquez, P. Salières, L. F. DiMauro, and F. Martín, Disentangling Spectral Phases of Interfering Autoionizing States from Attosecond Interferometric Measurements, Phys. Rev. Lett. 122, 253203 (2019).

[26] S. Beaulieu, A. Comby, A. Clergerie, J. Caillat, D. Descamps, N. Dudovich, B. Fabre, R. Géneaux, F. Légaré, S. Petit, B. Pons, G. Porat, T. Ruchon, R. Taïeb, V. Blanchet, and Y. Mairesse, Attosecond-resolved photoionization of chiral molecules, Science 358, 1288 (2017).

[27] M. R. F. Siggel, J. B. West, M. A. Hayes, A. C. Parr, J. L. Dehmer, and I. Iga, Shape-resonance-enhanced continuum- 
continuum coupling in photoionization of $\mathrm{CO}_{2}$, J. Chem. Phys. 99, 1556 (1993).

[28] R. R. Lucchese and V. McKoy, Studies of differential and total photoionization cross sections of carbon dioxide, Phys. Rev. A 26, 1406 (1982).

[29] R. R. Lucchese, Effects of interchannel coupling on the photoionization cross sections of carbon dioxide, J. Chem. Phys. 92, 4203 (1990).

[30] R. R. Lucchese and V. McKoy, Comparative studies of a shaperesonant feature in the photoionization of $\mathrm{CO}_{2}$, J. Phys. Chem. C 85, 2166 (1981).

[31] A. G. Harvey, D. S. Brambila, F. Morales, and O. Smirnova, An R-matrix approach to electron-photon-molecule collisions: Photoelectron angular distributions from aligned molecules, J. Phys. B: At., Mol., Opt. Phys. 47, 215005 (2014).

[32] P. M. Paul, E. S. Toma, P. Breger, G. Mullot, F. Augé, Ph. Balcou, H. G. Muller, and P. Agostini, Observation of a train of attosecond pulses from high harmonic generation, Science 292, 1689 (2001).

[33] H. G. Muller, Reconstruction of attosecond harmonic beating by interference of two-photon transitions, Appl. Phys. B 74 (Suppl 1), s17 (2002).

[34] Y. Mairesse and F. Quéré, Frequency-resolved optical gating for complete reconstruction of attosecond bursts, Phys. Rev. A 71, 011401(R) (2005).

[35] J. M. Dahlström, D. Guénot, K. Klünder, M. Gisselbrecht, J. Mauritsson, A. L'Huillier, A. Maquet, and R. Taïeb, Theory of attosecond delays in laser-assisted photoionization, Chem. Phys. 414, 53 (2013).

[36] I. Jordan, M. Huppert, S. Pabst, A. S. Kheifets, D. Baykusheva, and H. J. Wörner, Spin-orbit delays in photoemission, Phys. Rev. A 95, 013404 (2017).

[37] I. Jordan and H. J. Wörner, Extracting attosecond delays from spectrally overlapping interferograms, J. Opt. 20, 024013 (2018).

[38] T. N. Rescigno and A. E. Orel, Separable approximation for exchange interactions in electron-molecule scattering, Phys. Rev. A 24, 1267 (1981).

[39] T. N. Rescigno and B. I. Schneider, Disappearance of continuum exchange integrals from algebraic variational calculations of electron scattering, Phys. Rev. A 37, 1044(R) (1988).

[40] B. I. Schneider and T. N. Rescigno, Complex Kohn variational method: Application to low-energy electron-molecule collisions, Phys. Rev. A 37, 3749 (1988).

[41] A. E. Orel and T. N. Rescigno, Variational expressions for firstorder properties involving continuum wave functions, Phys. Rev. A 41, 1695 (1990).

[42] T. N. Rescigno and A. E. Orel, Continuum basis functions in the complex Kohn variational method, Phys. Rev. A 43, 1625 (1991).

[43] C. W. McCurdy and T. N. Rescigno, Beyond the primitive separable exchange approximation in electron-molecule scattering, Phys. Rev. A 46, 255 (1992).

[44] T. N. Rescigno, A. E. Orel, and C. W. McCurdy, Algebraic variational approach to atomic and molecular photoionization cross sections: Removing the energy dependence from the basis, Phys. Rev. A 55, 342 (1997).

[45] T. N. Rescigno and C. W. McCurdy, Improvements to the "standard" complex Kohn variational method: Towards the development of an "R-matrix theory without a box", in Novel Aspects of Electron-Molecule Collisions, edited by K. H. Becker (World Scientific Publishing, Singapore, 1998), p. 325.

[46] C. W. McCurdy, T. N. Rescigno, W. A. Isaacs, and D. E. Manolopoulos, Calculation of scattering amplitudes as continuous functions of energy: R-matrix theory without a box, Phys. Rev. A 57, 3511 (1998).

[47] W. Kohn, Variational methods in nuclear collision problems, Phys. Rev. 74, 1763 (1948).

[48] R. K. Nesbet, Analysis of the Harris variational method in scattering theory, Phys. Rev. 175, 134 (1968).

[49] R. K. Nesbet, Anomaly-free variational method for inelastic scattering, Phys. Rev. 179, 60 (1969).

[50] Electron-Molecule and Photon-Molecule Collisions, edited by T. N. Rescigno, V. McKoy, and B. I. Schneider (Plenum, New York, 1979).

[51] C. W. McCurdy, T. N. Rescigno, and B. I. Schneider, Interrelation between variational principles for scattering amplitudes and generalized R-matrix theory, Phys. Rev. A 36, 2061 (1987).

[52] J. Z. H. Zhang, S. I. Chu, and W. H. Miller, Quantum scattering via the S-matrix version of the Kohn variational principle, J. Chem. Phys. 88, 6233 (1988).

[53] T. N. Rescigno, C. W. McCurdy, A. E. Orel, and B. H. Lengsfield III, The complex Kohn variational method, in Computational Methods for Electron-Molecule Collisions, edited by W. M. Huo and F. A. Gianturco (Springer US, Boston, MA, 1995), p. 1.

[54] T. N. Rescigno, B. H. Lengsfield, and A. E. Orel, Interchannel coupling and ground state correlation effects in the photoionization of CO, J. Chem. Phys. 99, 5097 (1993).

[55] A. E. Orel and T. N. Rescigno, Photoionization of ammonia, Chem. Phys. Lett. 269, 222 (1997).

[56] S. Miyabe, C. W. McCurdy, A. E. Orel, and T. N. Rescigno, Theoretical study of asymmetric molecular-frame photoelectron angular distributions for C $1 s$ photoejection from $\mathrm{CO}_{2}$, Phys. Rev. A 79, 053401 (2009).

[57] H. Sann, T. Jahnke, T. Havermeier, K. Kreidi, C. Stuck, M. Meckel, M. S. Schöffler, N. Neumann, R. Wallauer, S. Voss, A. Czasch, O. Jagutzki, Th. Weber, H. Schmidt-Böcking, S. Miyabe, D. J. Haxton, A. E. Orel, T. N. Rescigno, and R. Dörner, Electron Diffraction Self-Imaging of Molecular Fragmentation in Two-Step Double Ionization of Water, Phys. Rev. Lett. 106, 133001 (2011).

[58] J. B. Williams, C. S. Trevisan, M. S. Schöffler, T. Jahnke, I. Bocharova, H. Kim, B. Ulrich, R. Wallauer, F. Sturm, T. N. Rescigno, A. Belkacem, R. Dörner, Th. Weber, C. W. McCurdy, and A. L. Landers, Imaging Polyatomic Molecules in Three Dimensions using Molecular Frame Photoelectron Angular Distributions, Phys. Rev. Lett. 108, 233002 (2012).

[59] N. Douguet, T. N. Rescigno, and A. E. Orel, Time-resolved molecular-frame photoelectron angular distributions: Snapshots of acetylene-vinylidene cationic isomerization, Phys. Rev. A 86, 013425 (2012).

[60] S. Marggi Poullain, C. Elkharrat, W. B. Li, K. Veyrinas, J. C. Houver, C. Cornaggia, T. N. Rescigno, R. R. Lucchese, and D. Dowek, Recoil frame photoemission in multiphoton ionization of small polyatomic molecules: photodynamics of $\mathrm{NO}_{2}$ probed by $400 \mathrm{~nm}$ fs pulses, J. Phys. B: At., Mol., Opt. Phys. 47, 124024 (2014). 
[61] S. Fonseca dos Santos, N. Douguet, A. E. Orel, and T. N. Rescigno, Ligand effects in carbon- $K$-shell photoionization, Phys. Rev. A 91, 023408 (2015).

[62] C. W. McCurdy, T. N. Rescigno, C. S. Trevisan, R. R. Lucchese, B. Gaire, A. Menssen, M. S. Schöffler, A. Gatton, J. Neff, P. M. Stammer, J. Rist, S. Eckart, B. Berry, T. Severt, J. Sartor, A. Moradmand, T. Jahnke, A. L. Landers, J. B. Williams, I. BenItzhak, R. Dörner, A. Belkacem, and Th. Weber, Unambiguous observation of F-atom core-hole localization in $\mathrm{CF}_{4}$ through body-frame photoelectron angular distributions, Phys. Rev. A 95, 011401(R) (2017).

[63] E. G. Champenois, L. Greenman, N. Shivaram, J. P. Cryan, K. A. Larsen, T. N. Rescigno, C. W. McCurdy, A. Belkacem, and D. S. Slaughter, Ultrafast photodissociation dynamics and nonadiabatic coupling between excited electronic states of methanol probed by time-resolved photoelectron spectroscopy, J. Chem. Phys. 150, 114301 (2019).

[64] A. U. Hazi, A. E. Orel, and T. N. Rescigno, Ab Initio Study of Dissociative Attachment of Low-Energy Electrons to $\mathrm{F}_{2}$, Phys. Rev. Lett. 46, 918 (1981).

[65] T. N. Rescigno, C. W. McCurdy, and B. I. Schneider, Accurate $A b$ Initio Treatment of Low-Energy Electron Collisions with Polyatomic Molecules: Resonant Electron-Formaldehyde Scattering, Phys. Rev. Lett. 63, 248 (1989).

[66] B. I. Schneider, T. N. Rescigno, B. H. Lengsfield III, and C. W. McCurdy, Accurate Ab Initio Treatment of Low-Energy Electron Collisions with Ethylene, Phys. Rev. Lett. 66, 2728 (1991).

[67] B. H. Lengsfield III and T. N. Rescigno, Electron-molecule close coupling with correlated target wave functions: Application to impact dissociation of $F_{2}$, Phys. Rev. A 44, 2913 (1991).

[68] T. J. Gil, C. W. McCurdy, T. N. Rescigno, and B. H. Lengsfield III, Polarization and correlation effects in elastic electron- $\mathrm{Li}_{2}$ scattering, Phys. Rev. A 47, 255 (1993).

[69] T. N. Rescigno, D. A. Byrum, W. A. Isaacs, and C. W. McCurdy, Theoretical studies of low-energy electron- $\mathrm{CO}_{2}$ scattering: Total, elastic, and differential cross sections, Phys. Rev. A 60, 2186 (1999).

[70] T. N. Rescigno, C. S. Trevisan, and A. E. Orel, Dynamics of Low-Energy Electron Attachment to Formic Acid, Phys. Rev. Lett. 96, 213201 (2006).

[71] H. Adaniya, B. Rudek, T. Osipov, D. J. Haxton, T. Weber, T. N. Rescigno, C. W. McCurdy, and A. Belkacem, Imaging the Molecular Dynamics of Dissociative Electron Attachment to Water, Phys. Rev. Lett. 103, 233201 (2009).

[72] D. S. Slaughter, A. Belkacem, C. W. McCurdy, T. N. Rescigno, and D. J. Haxton, Ion-momentum imaging of dissociative attachment of electrons to molecules, J. Phys. B: At., Mol., Opt. Phys. 49, 222001 (2016).

[73] T. N. Rescigno, C. S. Trevisan, A. E. Orel, D. S. Slaughter, H. Adaniya, A. Belkacem, M. Weyland, A. Dorn, and C. W. McCurdy, Dynamics of dissociative electron attachment to ammonia, Phys. Rev. A 93, 052704 (2016).
[74] See Supplemental Material at http://link.aps.org/supplemental/ 10.1103/PhysRevA.102.023118 for a more detailed description of the data and theory analysis.

[75] P. Hockett, E. Frumker, D. M. Villeneuve, and P. B. Corkum, Time delay in molecular photoionization, J. Phys. B: At., Mol., Opt. Phys. 49, 095602 (2016).

[76] K. Varjú, Y. Mairesse, B. Carré, M. B. Gaarde, P. Johnsson, S. Kazamias, R. López-Martens, J. Mauritsson, K. J. Schafer, PH. Balcou, A. L'huillier, and P. Salières, Frequency chirp of harmonic and attosecond pulses, J. Mod. Opt. 52, 379 (2005).

[77] Á. Jiménez-Galán, F. Martín, and L. Argenti, Two-photon finitepulse model for resonant transitions in attosecond experiments, Phys. Rev. A 93, 023429 (2016).

[78] W. F. Chan, G. Cooper, and C. E. Brion, The electronic spectrum of carbon dioxide. Discrete and continuum photoabsorption oscillator strengths (6-203 eV), Chem. Phys. 178, 401 (1993).

[79] H. Lischka, R. Shepard, F. B. Brown, and I. Shavitt, New implementation of the graphical unitary group approach for multireference direct configuration interaction calculations, Int. J. Quantum Chem. 20, 91 (1981).

[80] R. Shepard, I. Shavitt, R. M. Pitzer, D. C. Comeau, M. Pepper, H. Lischka, P. G. Szalay, R. Ahlrichs, F. B. Brown, and J.-G. Zhao, A progress report on the status of the COLUMBUS MRCI program system, Int. J. Quantum Chem. 34, 149 (1988).

[81] H. Lischka, R. Shepard, R. M. Pitzer, I. Shavitt, M. Dallos, T. Müller, P. G. Szalay, M. Seth, G. S. Kedziora, S. Yabushita, and Z. Zhang, High-level multireference methods in the quantumchemistry program system COLUMBUS: Analytic MR-CISD and MR-AQCC gradients and MR-AQCC-LRT for excited states, GUGA spin-orbit CI and parallel CI density, Phys. Chem. Chem. Phys. 3, 664 (2001).

[82] H. Lischka, T. Müller, P. G. Szalay, I. Shavitt, R. M. Pitzer, and R. Shepard, COLUMBUS-a program system for advanced multireference theory calculations, Wiley Interdiscip. Rev.: Comput. Mol. Sci. 1, 191 (2011).

[83] H. Lischka, R. Shepard, I. Shavitt, R. M. Pitzer, M. Dallos, Th. Müller, P. G. Szalay, F. B. Brown, R. Ahlrichs, H. J. Böhm, A. Chang, D. C. Comeau, R. Gdanitz, H. Dachsel, C. Ehrhardt, M. Ernzerhof, P. Höchtl, S. Irle, G. Kedziora, T. Kovar, V. Parasuk, M. J. M. Pepper, P. Scharf, H. Schiffer, M. Schindler, M. Schüler, M. Seth, E. A. Stahlberg, J.-G. Zhao, S. Yabushita, Z. Zhang, M. Barbatti, S. Matsika, M. Schuurmann, D. R. Yarkony, S. R. Brozell, E. V. Beck, J.-P. Blaudeau, M. Ruckenbauer, B. Sellner, F. Plasser, and J. J. Szymczak, Columbus, an ab initio electronic structure program, 2012, release 7.0.

[84] T. H. Dunning, Jr., Gaussian basis sets for use in correlated molecular calculations. I. The atoms boron through neon and hydrogen, J. Chem. Phys. 90, 1007 (1989).

[85] https://figshare.com/account/home\#/projects/63164.

[86] N. Douguet, B. I. Schneider, and L. Argenti, Application of the complex Kohn variational method to attosecond spectroscopy, Phys. Rev. A 98, 023403 (2018).

[87] U. Fano, Effects of configuration interaction on intensities and phase shifts, Phys. Rev. 124, 1866 (1961). 